\title{
Estado actual de la taxonomía de bivalvos dulceacuícolas chilenos: progresos y conflictos
}

\author{
State of the art in taxonomy of Chilean freshwater bivalves: advances and difficulties
}

\author{
ESPERANZA PARADA \& SANTIAGO PEREDO
}

\author{
Departamento de Ciencias Biológicas y Químicas, Facultad de Ciencias, Universidad Católica de \\ Temuco, Casilla 15-D, Temuco, Chile; e-mail: eparada@uct.cl
}

\begin{abstract}
RESUMEN
Se presenta una revisión actualizada de la taxonomía de los bivalvos dulceacuícolas chilenos. Los bivalvos descritos a la fecha, están adscritos a dos familias: Hyriidae representada sólo por el género Diplodon con dos especies $(D$. chilensis y D. solidulus) y Sphaeriidae representada por tres géneros: Pisidium con siete especies $(P$. chilense, $P$. magellanicum, $P$. lebruni, P. observationis, $P$. meierbrooki, $P$. huillichum y $P$. llanquihuense), Sphaerium con dos especies (S. lauricochae y $S$. forbesi) y Musculium con dos especies (M. argentinum y M. patagonicum). Entidades subgenéricas y subespecíficas son reconocidas sólo para Hyriidae. Se incluye la sinonimia, localidad tipo y distribución geográfica donde las especies han sido registradas. Los caracteres diagnósticos más confiables para la identificación de las especies de Hyriidae son la morfología de la charnela en adultos y en larvas, la presencia o ausencia de los dientes larvales, y la posición y forma de éstos. En los Sphaeriidae, los caracteres taxonómicos más confiables a nivel de género son la forma y el número de sifones, y el tamaño de ambas hemibranquias; a nivel específico, son la morfometría y morfología de las valvas, la que incluye, posición del umbo, características de la charnela, forma y tamaño de los dientes cardinales, esculpido de las valvas y densidad de poros en la superficie interna de ellas. Se discuten los resultados en relación a los progresos y conflictos respecto del ordenamiento taxonómico y sistemático en ambas familias, las dificultades para la recopilación de la información, la necesidad de redescribir algunas especies a la luz de nuevas técnicas citogenéticas y moleculares y ampliar la base de datos con nuevos registros en áreas geográficas no estudiadas aún. Se propone a Pisidium chilense, $P$. huillichum, P. llanquihuense y P. lebruni como especies endémicas de Chile y se bosquejan regiones zoogeográficas considerando la distribución de las especies de esféridos chilenos, registradas a la fecha.
\end{abstract}

Palabras clave: Hyriidae, Sphaeriidae, taxonomía, especies endémicas, regiones zoogeográficas, Chile.

\begin{abstract}
An update of the Chilean freshwater bivalve taxonomy is presented. The bivalves described at present are adscribed to two families: Hyriidae represented only by the genus Diplodon with two species (D. chilensis and D. solidulus) and Sphaeriidae represented by three genera: Pisidium with seven species ( $P$. chilense, P. magellanicum, $P$. lebruni, $P$. observationis, P. meierbrooki, $P$. huillichum and $P$. llanquihuense), Sphaerium with two species (S. lauricochae and S. forbesi), and Musculium with two species (M. argentinum and M. patagonicum). Sub-generic and sub-specific taxa are recognized for Hyriidae only. The present study includes the synonymy, type locality and geographic distribution where the species have been recorded. The most reliable diagnostic features for Hyriidae species identification are the hinge morphology in adult specimens, the presence or absence of the valve teeth, and their position and shape in larvae. At the generic level within Sphaeriidae, the most reliable taxonomic characters are the morphology and number of the siphons, and the size of both demibranchs. At the species level these characters are valve morphometry and morphology, which includes beak position, hinge features, shape and size of cardinal teeth, valve sculpture and pore density of the inner surface of valves. Results are discussed regarding advances and conflicts related to the taxonomic and systematic arrangement in both families, the difficulties in data compilation, the need to re-describe some species using cytogenetic and molecular techniques, and to increase this database with new records in geographic areas not yet studied. Pisidium chilense, $P$. huillichum, $P$. llanquihuense and $P$. lebruni are proposed as endemic species for Chile and zoogeographic regions are sketched based on the distribution of Chilean spheriids reported at the present.
\end{abstract}

Key words: Hyriidae, Sphaeriidae, taxonomy, endemic species, zoogeographical regions, Chile.

\section{INTRODUCCIÓN}

La creciente importancia que cobra el conocimiento y análisis de la biodiversidad, requiere a su vez, de una respuesta por parte de especialistas para conocer los grupos faunísticos presentes en un área geográfica dada, ya que, el catastro de especies es un importante punto de partida para la 
gran mayoría de los estudios relacionados con conservación de la biodiversidad así como para el posterior desarrollo de políticas de conservación (Ojeda 1998). Requerimientos del conocimiento científico y los cada vez más frecuentes estudios ambientales locales o regionales, relacionados con la presencia de especies, estados taxonómicos y de conservación de ellas, hacen de interés disponer de una lista que señale el status taxonómico actualizado de las especies. Las revisiones más completas realizadas en Chile respecto de su biodiversidad, muestran indiscutiblemente la falta de conocimiento respecto de algunos grupos faunísticos; tal es el caso de los moluscos dulceacuícolas y en especial la de los bivalvos dentro de aquellos. La ausencia total de antecedentes respecto de este grupo se ve reflejada en los reportes entregados por Lozada \& Osorio (1995). Revisiones más especializadas respecto del Phylum Mollusca llevadas a cabo por Osorio \& Bahamonde (1970) y Valdovinos (1999) demuestran que tras tres décadas, el avance del conocimiento de la biodiversidad ha sido aparentemente muy precario en relación a los bivalvos dulceacuícolas chilenos.

El estado actual del conocimiento de los bivalvos de agua dulce en Chile, permite señalar que a diferencia de lo que ocurre con los bivalvos marinos, los dulceacuícolas constituyen un grupo menos diverso. Los bivalvos presentes en Chile pertenecen a dos familias: Sphaeriidae que incluye las especies conocidas como almejas "píldora" ("pill" clams) y las almejas "uña de dedo" ("finger nail" clams), y la Familia Hyriidae que incluye los vulgarmente conocidos en Chile como choritos de agua dulce. Ambas han registrado a lo largo del tiempo serios conflictos taxonómicos, en especial los híridos, razón por la cual su conocimiento es precario. Muchos de estos conflictos se han suscitado debido a los cambios que ha experimentado el concepto de especie a través del tiempo, situación que también se ha presentado en bivalvos dulceacuícolas de otros continentes (Williams \& Mulvey 1997). Lo anterior constituyen limitantes que pueden comprometer los resultados de estudios limnológicos a la hora de reportar la presencia de especies y su distribución geográfica.

El objetivo de la presente comunicación es presentar una visión actualizada de la situación relativa a la taxonomía y sistemática de los bivalvos dulceacuícolas presentes en las aguas continentales de Chile considerando sus logros y conflictos y dar a conocer para las especies actualmente registradas, la sinonimia y la distribución geográfica donde han sido reportadas.

\section{MATERIALES Y MÉTODOS}

Los antecedentes presentados fueron obtenidos mediante revisiones bibliográficas, consultas a especialistas y material recolectado por los autores, el que se encuentra depositado en la Colección Museológica del Departamento de Ciencias Biológicas y Químicas de la Universidad Católica de Temuco, en el Museo Nacional de Historia Natural de Santiago de Chile y algunas especies en el Museo de La Plata, Buenos Aires, Argentina.

\section{RESULTADOS}

Los bivalvos descritos desde 1828 a la fecha, están adscritos a dos familias (Tabla 1): Hyriidae representada sólo por el género Diplodon con las especies D. chilensis y D. solidulus, y Sphaeriidae representada por tres géneros: Pisidium con las especies P. chilense, P. magellanicum, P. lebruni, $P$. observationis, $P$. meierbrooki, $P$. huillichum y $P$. llanquihuense; el género Sphaerium con las especies $S$. lauricochae y $S$. forbesi; y el género Musculium con las especies $M$. argentinum y $M$. patagonicum. Entidades subgenéricas y subespecíficas son reconocidas sólo para Hyriidae.

\section{Familia Hyridae}

La Familia Hyriidae se distribuye actualmente en América del Sur, Australia y Nueva Zelandia. En Chile, sólo está representada por la Subfamilia Hyriinae y la Tribu Diplodontini. Diplodon es el único género que habita los cuerpos de aguas continentales chilenos y está representado por dos subgéneros: Diplodon y Australis.

Diplodon (Diplodon) chilensis (Gray, 1828)

Localidad tipo: Lago Villarrica ( $\left.39^{\circ} 18^{\prime} \mathrm{S} ; 72^{\circ} 05^{\prime} \mathrm{O}\right)$, Novena Región de Chile.

Distribución geográfica: en Chile, los rangos de distribución geográfica norte-sur actualmente no están bien delimitados, aunque se ha señalado desde el norte de Chile hasta la Patagonia y Tierra del Fuego (Haas 1969, Peredo \& Parada 1984). En Argentina desde la Provincia de Mendoza hasta la Patagonia chileno-argentina (Bonetto 1973a). 
Diplodon (Diplodon) chilensis chilensis (Gray, 1828) (sensu Haas 1969)

Unio chilensis Gray, 1828

Unio smithi (Gray, 1834)

Unio obtusa (d'Orbigny, 1835)

Unio depressa (d'Orbigny, 1835)

Unio atratus (Sowerby, 1839)

Unio chilensis (Philippi, 1847)

Unio araucanus (Philippi, 1847)

Unio molinae (Philippi, 1847)

Diplodon molinae (Philippi, 1847)

Unio casablancae (Philippi, 1848)

Unio chiloensis (Kuster, 1856)

Unio gassiesi (Kuster, 1856)

Unio limense (Kuster, 1856)

Unio modestus (Kuster, 1856)

Unio fragilis (Sowerby, 1866)

Unio colchaguensis (Philippi, 1869)

Unio diplodon (Philippi, 1869)

Unio foncki (Philippi, 1869)

Unio jacobaeus (Philippi, 1869)

Unio ianthinus (Philippi, 1869)

Unio landbecki (Philippi, 1869)

Unio longus (Philippi, 1869)

Unio montanus (Philippi, 1869)

Unio valdivianus (Philippi, 1869)

Unio cuprinus (Simpson, 1900)

Diplodon aureus (Simpson, 1900)

Localidad tipo: Lago Villarrica $\left(39^{\circ} 18^{\prime} \mathrm{S}\right.$, $72^{\circ} 05^{\prime}$ O), Novena Región de Chile.

Distribución geográfica: registros actuales de los autores, la señalan desde la Séptima Región de Chile, en el Río Mataquito, Curicó (3458'S, $\left.71^{\circ} 48^{\prime} \mathrm{O}\right)$, hasta el Río Negro, Istmo de Ofqui (46³7’S; 74¹0’O), Undécima Región de Chile.

Diplodon (Diplodon) chilensis patagonicus (d'Orbigny, 1846 )( sensu Haas 1969)

Unio patagonica d'Orbigny, 1846

Diplodon frenzelli (Ihering, 1893)

Diplodon huapensis (Bartsch, 1906)

Localidad tipo: Río Negro, Argentina (Bonetto 1973b).

Distribución geográfica: ocupa preferentemente el área de la Patagonia chileno-argentina. Según Bonetto (1973a, 1973b) esta subespecie parece extenderse a toda la cadena de los lagos araucanos por el lado argentino, desde la Provincia de Mendoza hasta el Lago Futalauquén, adentrándose variablemente en algunos ríos como el Negro y el Neuquén. De acuerdo a Haas (19301931) podría incluir la Provincia de Buenos Ai- res. En Chile, no se ha registrado la presencia de esta subespecie en estas últimas décadas.

Diplodon (Australis) solidulus Philippi, 1869

Localidad tipo: Puerto Montt, Décima Región de Chile (Bonetto et al. 1986).

Distribución geográfica: Puerto Montt, Décima Región de Chile (Bonetto et al 1986).

\section{Familia Sphaeriidae}

La familia Sphaeriidae es de distribución mundial y está representada en Chile por tres géneros, también cosmopolitas (Tabla 1). El género

\section{TABLA 1}

Lista sistemática de los bivalvos dulceacuícolas de Chile

Systematic list of Chilean freshwater bivalves

Clase Bivalvia (= Pelecipoda, Lamellibranchiata)

Subclase Lamellibranchia

Superorden Heterodonta (= Eulamellibranchia)

Orden Paleoheterodonta

Superfamilia Unionacea

Familia Hyriidae

Subfamilia Hyriinae

Género Diplodon (Spix, 1827)

Subgénero Diplodon ss (Spix, 1827)

Especie Diplodon (Diplodon) chilensis (Gray, 1828)

Subespecie Diplodon (Diplodon) chilensis chilensis (Gray, 1828)(sensu Haas 1969)

Diplodon (Diplodon) chilensis patagonicus (d'Orbigny, 1846)(sensu Haas 1969)

Subgénero Australis Bonetto, Tassara y Rumi,1986

Especie Diplodon (Australis) solidulus Philippi, 1869

Orden Veneroida

Superfamilia Sphaeriacea

Familia Sphaeriidae

Género Pisidium Pfeiffer, 1821

Especie: Pisidium chilense (d'Orbigny,1846) Pisidium magellanicum (Dall, 1908)

Pisidium lebruni Mabille, 1884

Pisidium observationis Pilsbry, 1911

Pisidium meierbrooki Kuiper y Hinz, 1983

Pisidium huillichum Ituarte, 1999

Pisidium llanquihuense Ituarte,1999

Género Sphaerium Scopoli, 1777

Especie Sphaerium lauricochae (Phillipi, 1869)

Sphaerium forbesi (Phillipi,1869)

Género Musculium Link, 1807

Especie Musculium argentinum (d'Orbigny, 1835) Musculium patagonicum Pilsbry, 1911 
Pisidium está actualmente representado por siete especies distribuidas a lo largo del territorio nacional. El género Sphaerium está representado a la fecha por sólo dos especies ocupando el área altoandina, compartiendo territorio con Perú y Bolivia. El género Musculium, también representado por dos especies, ocupan la región centro-sur y austral de Chile, compartiendo áreas de distribución con Argentina.

Pisidium chilense (d'Orbigny, 1846)

Cyclas chilense d'Orbigny, 1846

Localidad tipo: Concepción, Octava Región de Chile (36 $50^{\circ}$ 'S, $72^{\circ} 50^{\prime} \mathrm{O}$ ) sensu d'Orbigny (1846). De acuerdo a Gray (1855) la localidad tipo sería

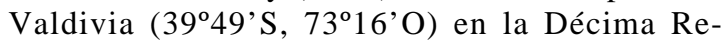
gión de Chile (Ituarte 1999).

Distribución geográfica: Concepción, Octaba Región de Chile; Valdivia y Chaitén, Décima Región de Chile (Ituarte 1999). Recientemente los autores han colectado especímenes en el río Cautín, a la altura de la ciudad de Lautaro $\left(38^{\circ} 32^{\prime} \mathrm{S}, 7^{\circ} 26^{\prime} \mathrm{O}\right)$ y lago Villarrica $\left(39^{\circ} 18^{\prime} \mathrm{S}\right.$, $\left.72^{\circ} 05^{\prime} \mathrm{O}\right)$, Novena Región de Chile (E. Parada, S. Peredo, C. Sobarzo \& P. Jara-Seguel, resultados no publicados)

Pisidium magellanicum (Dall 1908)

Corneocyclas magellanicus Dall 1908.

Localidad tipo: Estrecho de Magallanes (Ituarte 1996).

Distribución geográfica: en Chile se encuentra entre $\operatorname{los} 45^{\circ}$ y $55^{\circ} \mathrm{S}$ (Valdovinos 1999) y río Chico, río Blanco en el $48^{\circ} \mathrm{S}$ (Provincia de Santa Cruz) y lago Amutui-Quimei (Provincia de Chubut) en Argentina (Ituarte 1996).

\section{Pisidium lebruni Mabille, 1884}

Localidad tipo: Cabeza del Mar, (52 $47^{\circ} \mathrm{S}$, $\left.71^{\circ} 05^{\prime} \mathrm{O}\right)$ Punta Arenas, Duodécima Región de Chile.

Distribución geográfica: Punta Arenas, Duodécima Región de Chile. De acuerdo a Ituarte (1995), esta especie se conoce sólo en Punta Arenas.

Pisidium observationis Pilsbry, 1911

Localidad tipo: Monte Observación (50²2'S, $68^{\circ} 57^{\prime}$ O) Provincia de Santa Cruz, Argentina.
Distribución geográfica: Argentina: provincias de Santa Cruz, Neuquén y Tierra del Fuego. Puerto Natales y Punta Arenas, Duodécima Región de Chile (Ituarte \& Gordillo 1991, Ituarte 1995).

Pisidium meierbrooki Kuiper \& Hinz, 1983

Localidad tipo: $7 \mathrm{~km}$ de la localidad de Batallas sobre la ruta La Paz-Tiquina, Bolivia.

Distribución geográfica: área alto andina de Bolivia, Perú y Chile (Kuiper \& Hinz 1983, Ituarte 1995).

Pisidium huillichum Ituarte, 1999

Localidad tipo: Lago Yelcho, Puerto Cárdenas ( $\left.43^{\circ} 10^{\prime} \mathrm{S}, 72^{\circ} 30^{\prime} \mathrm{O}\right)$, Provincia de Palena, Décima Región de Chile y Lago Llanquihue, Puerto Octay Provincia de Llanquihue, Décima Región de Chile.

Distribución geográfica: Décima Región de Chile (Ituarte 1999).

Pisidium llanquihuense Ituarte, 1999

Localidad tipo: Lago Llanquihue, Puerto Octay (entre $40^{\circ} 38^{\prime} \mathrm{S}, 72^{\circ} 31^{\prime} \mathrm{O}$ y $41^{\circ} 20^{\prime} \mathrm{S}, 73^{\circ} 0$ 'O) Décima Región de Chile y en río Notuco, Isla Grande de Chiloé, Décima Región de Chile (Ituarte 1999).

Distribución geográfica: Décima Región de Chile hasta Chiloé.

Sphaerium lauricochae (Philippi, 1869)

Cyclas lauricochae Philippi, 1869

Pisidium lauricochae (Philippi, 1869)

Localidad tipo: Lago Lauricocha, en la naciente del río Marañón, Perú (Ituarte 1995).

Distribución geográfica: área alto andina de Bolivia, Perú y Chile. En Chile, en ojos de Ascotán, provincia de Antofagasta a $3.800 \mathrm{~m}$ de altitud (Kuiper \& Hinz 1983, Ituarte 1995).

Sphaerium forbesi (Philippi, 1869)

Cyclas forbesii Philippi, 1869

Pisidium boliviense (Philippi, 1869)

Localidad tipo: Bolivia alta (Ituarte 1995).

Distribución geográfica: área alto-andina de Bolivia, Perú y Chile; ha sido citado además para Colombia (Kuiper \& Hinz 1983). En Chile, en 
Cotacotaní (Ituarte 1995), área cercana a varias localidades citadas por Kuiper \& Hinz (1983).

\section{Musculium argentinum (d'Orbigny, 1835)}

Cyclas argentina d'Orbigny, 1835

Sphaerium argentinum (d'Orbigny, 1835)

Localidad tipo: arroyo Pantanoso, Montevideo, Uruguay (Figueiras 1965).

Distribución geográfica: Ituarte (1996) reporta su presencia en Uruguay (Montevideo) y Argentina (provincias de Mendoza, Buenos Aires y Tierra del Fuego). En Chile ha sido reportado recientemente en el río Cautín (Novena Región de Chile) a la altura de la ciudad de Lautaro (38 $32^{\circ} \mathrm{S}$, $72^{\circ} 26^{\prime}$ O) por Sobarzo et al. (en prensa).

\section{Musculium patagonicum Pilsbry, 1911}

Sphaerium (Musculium) patagonicum Pilsbry, 1911 (sensu Valdovinos 1999)

Localidad tipo: río Chico, Provincia de Santa Cruz, Argentina (48 $\left.46^{\circ} \mathrm{S}, 70^{\circ} 22^{\prime} \mathrm{O}\right)$.

Distribución geográfica: en Argentina desde la Provincia de Santa Cruz hasta la Provincia de Neuquén por el norte. En Chile, Valdovinos (1999) señala una distribución entre $\operatorname{los} 50^{\circ}$ y $55^{\circ} \mathrm{S}$.

\section{DISCUSIÓN}

Antecedentes sobre el origen de bivalvos dulceacuícolas en el territorio chileno sólo existen respecto de los Hyriidae a partir del Eoceno. Parodiz (1977) señala que la superfamilia Unionacea pertenece a un grupo de origen norteamericano. De acuerdo a Parodiz (1977), los unionáceos habrían emigrado hacia el sur a principios del Terciario, simultáneamente con los mamíferos primitivos que poblaron la Patagonia. Los más antiguos ancestros de los unionáceos sudamericanos estuvieron representados por los Hyriidae en el Triásico en Pensylvania y Texas, grupo actualmente extinto en Norteamérica. Por otro lado, Graf \& Ó Foighil (2000) señalan que resultados de estudios filogenéticos realizados en híridos de Australia, Nueva Zelandia y Sudamérica son consistentes con la teoría del "puente terrestre" según el cual los Hyriidae surgieron en Australia y llegaron a Sudamérica a través de la Antártida. En Sudamérica los registros fósiles más antiguos corresponden al género Diplodon. En Argentina se han registrado en el Paleoceno, Mioceno, Plioceno y en el Pleistoceno (Parodiz \&
Bonetto 1963). También se han registrado fósiles de Diplodon en Perú y Ecuador en el Plioceno (Parodiz \& Bonetto 1963). En Chile han sido registrados en el Eoceno y en forma más reciente Dillehay (1984) y Sánchez \& Inostroza (1985) informan del hallazgo de valvas de Diplodon en asentamientos indígenas en el sur de Chile a fines del Pleistoceno.

Actualmente, el género Diplodon ocupa un amplio rango de distribución geográfica en América del Sur, existiendo diversas especies en Venezuela, Perú, Brasil, Argentina, Uruguay y Chile. En Chile, y especialmente en el centro y sur tanto ríos como lagos están colonizados por individuos de Diplodon (Peredo \& Parada 1986, Parada et al. 1989a, 1989b, 1990). Parada \& Peredo (1994) señalan que las poblaciones de río debieron ser las poblaciones primitivas de acuerdo a lo reportado por Dillehay (1984) y Sánchez \& Inostroza (1985). Posteriormente al originarse los lagos por procesos de deglaciación o actividad volcánica, surgen nuevos nichos que fueron ocupados por estos bivalvos gracias a su dispersión por peces que son los hospederos de las larvas gloquidias, por tanto, las poblaciones lénticas podrían ser consideradas como poblaciones secundarias.

Desde sus inicios, el ordenamiento taxonómico de los híridos sudamericanos y en especial las especies del género Diplodon (Spix, 1827) ha sido controvertido por cuanto cinco clasificaciones han sido propuestas a la fecha: Simpson 1914, Ortman 1921, Haas 1930-1931, Modell 19421943 y la más reciente de Parodiz \& Bonetto 1963 (Parodiz \& Bonetto 1963). De acuerdo a Parodiz \& Bonetto (1963) los unionáceos sudamericanos pertenecen a la familia Hyriidae, la cual también puede encontrarse en Australia y Nueva Zelandia, pero está ausente en el resto del mundo. Las formas sudamericanas pertenecen a la subfamilia Hyriinae la que está dividida en tres tribus: Diplodontini, Castaliini y Prisodontini. Diplodontini, formada mayoritariamente por el género Diplodon, está más estrechamente relacionada con las formas de Australia y Nueva Zelandia. La separación a nivel de subgénero ha sido infructuosa debido a los caracteres que se han utilizado, muchos de ellos morfológicos de gran variabilidad en los individuos y a la incorporación de procesos biológicos no tan detectables a simple vista como las características del ciclo de vida de los individuos. Es así como Parodiz \& Bonetto (1963) han distinguido dos entidades a nivel subgenérico: Diplodon ss, con larva parásita gloquidia y Rhipidodonta con gloquidia no parásita y desarrollo directo. Bonetto et al. (1986) agregan un nuevo subgénero, Australis, en aten- 
ción al punto de inserción ventral media de los dientes larvales de la gloquidia.

Los caracteres diagnósticos que se han utilizado para discriminar entre las especies del género Diplodon han sido también muy variados, siendo objeto de fuertes discusiones. Esto ha generado las constantes proposiciones nomenclaturales incluyendo a aquellas poblaciones que habitan en Chile. Se ha señalado que esta confusa situación taxonómica se debería, en primer lugar, a la variabilidad morfológica intra e inter-poblacional existente entre las poblaciones, principalmente, entre aquellas que habitan ríos y lagos, como lo han señalado Parada \& Peredo (1994) para poblaciones del centro sur de Chile. En segundo lugar, la confusión taxonómica se debería al escaso número de ejemplares utilizados y a algunos de los caracteres utilizados para la descripción. Los caracteres diagnósticos a nivel de adultos han sido la morfología y morfometría de la concha y la ubicación de la bolsa marsupial en la hemibranquia interna de la hembra y a nivel larval, la morfología y morfometría de la concha de la larva gloquidio, y la forma, posición y tamaño del diente larval (Parada et al. 1989a).

De los caracteres señalados anteriormente, se ha podido establecer que algunos constituyen un buen carácter taxonómico. Así, en los adultos los caracteres taxonómicos confiables serían fundamentalmente las características de la charnela, no así lo relativo a la biometría de la concha ni la posición de la bolsa marsupial en las hembras. En la larva gloquidio, el carácter taxonómico más confiable es la presencia o no de diente larval, y la posición y forma de éste (Parada et al. 1989a).

En Chile y la Patagonia argentina, y en base a todos los criterios antes mencionados, se han descrito aproximadamente 25 formas del género Diplodon, muchas de ellas descritas por Philippi (1847, 1848, 1869). Según Haas (1969) estas formas pueden subordinarse prácticamente en su totalidad a la especie Diplodon chilensis (Gray, 1828 ) y a dos subespecies $D$. ch. chilensis y $D$. ch. patagonicus. Estudios realizados por los autores dan cuenta de la presencia de $D$. ch. chilensis desde el río Mataquito ( $\left.34^{\circ} 58^{\prime} \mathrm{S}, 71^{\circ} 48^{\prime} \mathrm{O}\right)$ en la Séptima Región de Chile, hasta el río Negro, Istmo de Ofqui $\left(46^{\circ} 37^{\prime} \mathrm{S}, 74^{\circ} 10^{\prime} \mathrm{O}\right)$, Undécima Región de Chile. Diplodon ch. patagonicus habitaría la región más austral de Chile y la Patagonia argentina. Para esta última subespecie no se han reportado sitios precisos de colecta en Chile posteriores a lo señalado por Haas (1969). De acuerdo a lo anterior, se hace necesario llevar a cabo prospecciones en la región patagónica chilena a fin de confirmar o invalidar la presencia de ella.
Otro aspecto aún no dilucidado respecto de las especies de Diplodon en Chile atañe a la especie $D$. atratus. Bonetto (1961) alude a ella al analizar gloquidios adheridos a una valva registrada con el lote $\mathrm{N}^{\circ} 85628$ de la colección del U.S. National Museum, rotulados bajo el nombre de Chile como lugar de procedencia. De acuerdo a Bonetto (1961) esta especie estaría presente en Perú y Chile. Esta confusa situación ha hecho suponer la existencia de poblaciones de Diplodon a lo largo de todo Chile (Arica a Estrecho de Magallanes), como lo señalaran Peredo \& Parada (1984), quienes siguiendo a Haas (1969) la determinaron como $D$. chilensis. Es probable que D. atratus sea una de las tantas sinonimias de $D$. chilensis de acuerdo al análisis de gloquidias llevado a cabo por Parada et al. (1989 a) y por Parada \& Peredo (1994) procedente de diferentes poblaciones chilenas. Por otra parte, el análisis de la morfometría y morfología de las valvas y la ubicación de la bolsa marsupial en las hemibranquias internas de las hembras (Parada et al. 1989a) corroboran lo señalado anteriormente en el sentido que $D$. atratus sea sinónimo de $D$. chilensis. El material utilizado por Peredo \& Parada (1984, 1986), por Parada et al. (1989a, 1989b, 1990) y por Parada \& Peredo (1994) se encuentra depositado en la Colección Museológica del Departamento de Ciencias Biológicas y Químicas de la Universidad Católica de Temuco. Valvas de especímenes adultos de $D$. chilensis de las poblaciones estudiadas fueron enviadas a la Sección de Malacología del Museo Nacional de Historia Natural, Santiago de Chile.

Por otra parte, y en contraposición a lo señalado por Haas (1969), Bonetto et al. (1986) han descrito a $D$. solidulus con material proveniente de Puerto Montt; ambas propuestas incluyen poblaciones localizadas en el área de distribución geográfica correspondiente a $D$. chilensis chilensis. Bonetto et al. (1986) describe a Diplodon solidulus como perteneciente al nuevo subgénero Australis, a diferencia de $D$. chilensis que está adscrito al subgénero Diplodon. Cabe señalar que esta nueva proposición está basada en el estudio de un número reducido de ejemplares obtenidos del área de Puerto Montt, sin entregar antecedentes de su ubicación geográfica exacta, lo que hace dudar la validez de la propuesta mientras no se re-estudie ese material o se confirme su existencia con nuevas colectas.

Así como Haas (1969) nominó como Diplodon chilensis a la gran mayoría de las especies de híridos descritas para Chile, lo que ha sido corroborado con estudios bioecológicos por Parada et al. (1989a) y estudios cariológicos por Jara-Seguel et al. (2000) y por S. Peredo, P. Jara-Seguel, E. Parada y C. Palma-Rojas (resultados no publicados), situación similar ha ocurrido con la especie 
Quadrula asperata cuyos taxa originales fueron descritos por Isaac Lee entre 1827 y 1847 como Unio keineriana, $U$. asperatus y $U$. cahabensis (Williams \& Mulvey, 1997). De igual modo Williams \& Mulvey (1997) señalan que Johnson en 1970, incluyó 102 sinónimos para Elliptio complanata, lo que fue confirmado posteriormente con estudios citogenéticos y análisis multivariados de la morfología de la concha y las partes blandas realizados por Davis \& Mulvey (1993). Estos ejemplos de cambios taxonómicos que están apareciendo en la literatura de las almejas de agua dulce, son un reflejo de los cambios que ha ido experimentando el concepto de especie así como del uso de nuevas metodologías de análisis en el ámbito de la sistemática y taxonomía.

A diferencia de los Hyriidae, los Sphaeriidae no cuentan con antecedentes paleontológicos o evidencias en el territorio chileno que permitan elucubrar sobre su origen. De acuerdo a Keen \& Dance (1969), los esféridos aparecieron inicialmente en hábitat dulceacuícolas en el Cretácico. Actualmente alcanzan una distribución cosmopolita y ubicua (Clarke 1973, Burch 1975, Parodiz 1977, Kuiper 1983, Morton 1985, Korniushin 2000, Park \& Ó Foighil 2000). A pesar que los esféridos también se han reportado para Australia y Nueva Zelanda, aún no se han podido establecer afinidades con las especies chilenas como ocurre con los híridos. Actualmente en Chile los Sphaeriidae están representados por los tres géneros tradicionalmente adscritos a la familia Sphaeriidae: Pisidium, Sphaerium y Musculium los que presentan una distribución a nivel mundial.

El ordenamiento taxonómico y sistemático de los esféridos no está exento de contradicciones. Una de las clasificaciones modernas y más aceptadas incluye a la familia Sphaeriidae ("pill clams" y "fingernail clams") dentro del orden Veneroida y la superfamilia Sphaeriacea junto con las familias Corbiculidae ("basket clams") y Dreissenidae ("zebra mussels") (Park \& Ó Foighil 2000). Las formas chilenas reportadas a la fecha, están adscritas sólo a la familia Sphaeriidae. En los taxa inferiores también han existido controversias. Al respecto, muchos autores consideran que Sphaerium y Pisidium pertenecen a la familia Sphaeriidae (Kuiper 1983, Valdovinos 1999, Korniushin 2000, Park \& Ó Foighil 2000), mientras que Starobogatov (1977) los adscribe a la familia Pisididae. Baker (1927) y Clarke (1981) los adscriben a dos subfamilias, en tanto que Bowden \& Heppell (1968) y Ellis (1978) lo hacen a las familias Sphaeriidae y Pisididae respectivamente. El análisis realizado por Kuiper (1983) señala que hay argumentos en pro y en contra en cada una de las proposiciones.
La tendencia más aceptada es incluir a ambos géneros en la familia Sphaeriidae.

A nivel genérico y subgenérico, los caracteres taxonómicos más adecuados según Kuiper (1983) serían las características de la concha y en caso de incertidumbre las partes blandas son de utilidad, siendo imposible la identificación de animales sin sus valvas. En la actualidad la descripción de los especímenes incluye la morfometría y morfología de las valvas, forma y posición del umbo, características de la charnela, posición y forma del ligamento, forma y desarrollo de los dientes cardinales, el esculpido de las valvas y la densidad de poros de la superficie interna de ellas. En relación a las partes blandas, los caracteres taxonómicos para discriminar a nivel genérico dicen relación con las características y número de los sifones y con las características y grado de reducción de las hemibranquias (Korniushin 1996, 1997, 1998, $1999^{1}$, C.F. Ituarte comunicación personal). Las especies de Sphaerium y Musculium tienen ambos sifones, el branquial y el anal bien desarrollados y ambas hemibranquias están presentes las que se modifican para generar los sacos incubadores. Según C.F. Ituarte (comunicación personal) ha sido bastante discutida la real existencia de ambos géneros. Según las ideas más conservadoras, la diferencia fundamental radica en que Musculium tiene ambos sifones bien desarrollados aunque fusionados en casi la totalidad de su longitud, sólo separados en el extremo; en tanto en las especies de Sphaerium, ambos sifones están sólo unidos en su base y bien separados en el resto de su longitud. Korniushin (1998) señala que la diferencia entre ambos géneros radica en ciertas características del nefridio, ya que Musculium presenta un saco excretor pequeño y embudo delgado y alargado sin válvula interna. Siguiendo los criterios de Korniushin (1998), C.F. Ituarte (comunicación personal) señala que correspondería incluir a Sphaerium lauricochae y $S$. forbesi en el género Musculium, mientras que Musculium argentinum y M. patagonicum estarían correctamente asignados al género Musculium. Siguiendo el criterio anterior, la especie aludida por Valdovinos (1999) debería registrarse sólo como $M$. patagonicum Pilsbry, 1911.

${ }^{1}$ KORNIUSHIN AV (1999) Significance of gill characters for taxonomy in Sphaeriidae (Eulamellibranchiata) and some other bivalve groups. Biology \& Evolution of the Bivalvia. An International Meeting to focus solely on the Bivalvia. Malacological Society of London: 14-17. September 1999, London, United Kingdom. R 40. 
Respecto de las especies adscritas al género Pisidium, éstas pueden presentar una o dos hemibranquias así como un sifón (el anal) o los dos (branquial y anal). Esta situación se ha observado en la fauna sudamericana y chilena (C.F. Ituarte comunicación personal), así como en la existente en otras regiones del hemisferio norte (Korniushin 1998). Este hecho dificulta aún más la identificación de los ejemplares. Estudios realizados por Korniushin $\left(1999^{1}\right)$ relacionados con la ontogenia de las ctenidia señala que la posición de la hemibranquia externa no cambia durante la ontogenia pero que el tiempo de aparición de la hemibranquia externa y su tasa de crecimiento varía significativamente entre las especies y entre los géneros, y por tanto esta característica podría considerarse también como un carácter taxonómico. Lo significativo de este estudio es que demuestra la confusión existente respecto de la taxonomía a nivel genérico, ya que estudiando la ontogenia de las ctenidia en Sphaerium, se observó que éste presentaba un estado ontogenético unilamelar similar a lo registrado para Pisidium. Tal como ha estado ocurriendo con los híridos, el uso de técnicas citogenéticas y moleculares podrían aportar a futuros nuevos antecedentes taxonómicos y sistemáticos sobre este grupo de bivalvos dulceacuícolas.

A pesar de los conflictos taxonómicos antes señalados, un avance significativo en estas dos últimas décadas se ha observado respecto del conocimiento de la biodiversidad de los bivalvos dulceacuícolas chilenos. Aún cuando la gran mayoría de los trabajos llevados a cabo en Chile por los autores del presente trabajo en estas últimas décadas no han estado enmarcados en el ámbito de la taxonomía y de la sistemática, su aporte ha sido esencial en el conocimiento de la diversidad específica existente, permitiendo de paso aclarar algunos conflictos taxonómicos que se arrastran desde la primera mitad del siglo XX. Actualmente en los híridos existen criterios taxonómicos morfológicos confiables tanto a nivel de adulto como larval que permiten identificar fehacientemente una población. El conocimiento del cariotipo de $D$. chilensis $(2 \mathrm{n}=34)$ reportado por Jara-Seguel et al. (2000), así como estudios citogenéticos en $D$. chilensis reportados por Jara-Seguel et al. (2000) $)^{2}$, Parada et al. $(2000)^{3}$ y S. Peredo, P. Jara- Seguel, E. Parada y C. Palma-Rojas (resultados no publicados) han contribuido significativamente a dilucidar y confirmar lo señalado por Haas (1969) y Parada et al. (1989 a) respecto a aquellos conflictos producidos a finales del siglo XIX y comienzos del XX, producto de la plasticidad fenotípica que evidencian las poblaciones que habitan ambientes diferentes (Parada et al. 1989a, 1989b, Parada \& Peredo
1994). Esta situación llevó a Philippi (1847, 1848, 1869) a describir más de 20 especies, todas las cuales están adscritas a $D$. chilensis en la actualidad. De igual modo, estudios a nivel molecular llevados a cabo por Graf \& Ó Foighil (2000) que incluyeron a $D$. chilensis están aportando a un mayor conocimiento de la sistemática y filogenia de este grupo faunístico.

Respecto de los esféridos, el aumento en el conocimiento de su diversidad en Chile ha sido gracias a los aportes de Ituarte $(1995,1996,1999)$ y Sobarzo et al. (en prensa), quienes han utilizado todos los criterios taxonómicos validados actualmente. En el caso de las dos nuevas especies de Pisidium descritas por Ituarte (1999) con presencia en Chile, $P$. huillichum y P. llanquihuense, cuya distribución geográfica es muy similar, surge la duda de la validez de estas especies. Sin embargo, la descripción que el autor hace de cada una de ellas, relacionado con el tamaño de los especímenes, la morfología y morfometría de las valvas, el tipo de ligamento interno, el esculpido externo de las valvas, el número de embriones en el saco incubatriz, las características de las aberturas sifonales, entre otras, además de los correspondientes registros fotográficos del trabajo citado permiten reconocerlas como entidades diferentes, distinguir ambas especies entre sí y de las otras especies de Pisidium descritas para Chile. Lo anterior lleva a los autores del presente trabajo a validar a la luz del material disponible a ambas especies citadas para Chile. Sin embargo, estudios futuros citogenéticos o moleculares podrían confirmar o invalidar lo propuesto por Ituarte (1999). No obstante lo anterior y aun cuando la diversidad específica de este grupo

\section{${ }^{2}$ JARA-SEGUEL P, S PEREDO, E PARADA \& C PALMA-ROJAS (2000) Citogenética descriptiva y comparada entre poblaciones alopátricas de Diplodon chilensis (Gray 1828)(Bivalvia: Hyriidae). Sixth International Congress on Medical and Applied Malacology, International Society for Medical and Applied Malacology, 4-8 September, 2000, Havana, Cuba: 32-33.}

\footnotetext{
${ }^{3}$ PARADA E, S PEREDO, P JARA-SEGUEL, C PALMA-ROJAS \& G LARA (2000) Una aproximación desde la citogenética al conocimiento de la historia evolutiva y sistemática de los hyriidos chilenos. Sixth International Congress on Medical and Applied Malacology, International Society for Medical and Applied Malacology, 4-8 September, 2000, Havana, Cuba: 44-45.
} 
es mayor que la de los híridos, el estado del conocimiento ha estado centrado en descripciones de nuevas especies, revisiones y/o registro de especies, faltando muchas áreas geográficas aún por muestrear, así como otros estudios que permitan conocer acerca de la ecología (dinámica poblacional, densidad, rol en el ecosistema que integran), biología reproductiva, ciclos de vida, y otros, que permitan un mayor conocimiento de cada una de las especies existentes.

El conocimiento que se tiene a la fecha de los bivalvos dulceacuícolas tanto de híridos, pero muy especialmente de esféridos no permiten aún configurar un mapa acabado de la distribución geográfica de las especies. Varios hechos pueden modificar una posible proposición. Una de ellas es que a la fecha los antecedentes de registros de especies que se disponen provienen de estudios en áreas geográficas puntuales faltando un registro a lo largo y ancho del territorio chileno. Por otro lado, si se consideran los mecanismos de dispersión tanto de híridos como de esféridos, son éstos últimos los que pueden hacer cambiar sustancialmente el panorama. Los híridos se dispersan fundamentalmente a través de los peces dada la condición parásita de la larva gloquidio pudiendo colonizar fácilmente diferentes áreas geográficas dentro de una misma hoya hidrográfica independiente del sustrato que exista (observación personal de los autores). También lo pueden hacer, pero en menor proporción, de un cuerpo de agua a otro por medio de las aves, quienes acarrean almejas adheridas a sus patas (Morgan 1982), situación no documentada para Chile. Los esféridos han evidenciado una dispersión pasiva a través del acarreo de individuos adheridos a tarsos o antenas de insectos o plumas de aves acuáticas (Rees 1965, Morgan 1982). Sin embargo, los individuos de esféridos requieren de un sustrato fundamentalmente del tipo areno-fangoso para su sobrevivencia, de acuerdo a las observaciones personales de los autores en terreno. La dispersión y el asentamiento se ve favorecida en el caso de los híridos por la condición de incubación branquial de las hembras y en esféridos por su condición hermafrodita y autofecundación en condiciones menos favorables (Heard 1965, Meier-Brook 1970) permitiendo la formación en poco tiempo de una nueva población.

No obstante lo anterior, el análisis de la distribución geográfica de los esféridos chilenos permite bosquejar algunas regiones zoogeográficas. Al respecto, Pisidium meierbrooki, Sphaerium lauricochae y $S$. forbesi serían especies propias de la región alto-andina compartiendo áreas geográficas con Perú y Bolivia. Especies propias de regiones patagónicas que comparten áreas geográficas con Argentina serían Pisidium magellanicum, $P$. observationis y Musculium patagonicum. Pisidium lebruni, también es una especie patagónica, pero registrada actualmente sólo para Chile. En el centro sur de Chile se han registrado Pisidium chilense, P. huillichum, P. llanquihuensis y Musculium argentinum. Las tres especies de Pisidium sólo han sido registradas en Chile, no así $M$. argentinum que es de amplia distribución en el cono sur de Sudamérica. Lo anterior lleva a los autores a proponer la existencia de tres áreas zoogeográficas definidas de presencia de esféridos y a la postulación de cuatro especies endémicas para Chile, propuesta que es inicial y que se requiere de estudios futuros para su corroboración, en especial establecer su presencia en áreas geográficas aún no estudiadas, entre los paralelos 18 y $35^{\circ} \mathrm{S}$ en particular y corroborar la presencia o ausencia de $P$. lebruni en Argentina.

Respecto de los híridos, la baja diversidad específica, no amerita un análisis mayor. Con los datos existentes se puede señalar ausencia de endemismo, pero se hace necesario estudiar los cuerpos de aguas continentales de la zona norte y extremo sur de Chile a fin de tener claridad de la distribución de las especies y subespecies actualmente validadas para Chile.

La revisión del estado actual de la sistemática y taxonomía que se ha presentado constituye un aporte para avanzar en el conocimiento de la biodiversidad chilena. Como se ha podido apreciar parte importante de la información que se conoce de los bivalvos dulceacuícolas chilenos se halla dispersa en diversos trabajos científicos realizados especialmente por extranjeros quienes obtuvieron el material al efectuar expediciones que han recorrido nuestro territorio lo que ha llevado a que prácticamente todos los tipos se encuentran depositados en distintos museos fuera del país, situación que ha dificultado enormemente el análisis de este material. Por lo anterior, se hace necesario redescribir nuevamente algunas especies a la luz de la información existente utilizando los caracteres taxonómicos ampliamente aceptados y complementada con las opciones metodológicas y tecnológicas que la taxonomía y la sistemática actualmente dispone. Los autores, en alguna medida comparten lo señalado por Kuiper (1983) respecto de que una manera útil y confiable de identificar un espécimen es hacerlo por comparación con ejemplares válidamente identificados en colecciones públicas. De ahí la importancia de mantener y disponer de colecciones confiables y completas en nuestro país de moluscos bivalvos dulceacuícolas presentes en Chile. En este propósito, es importante el compromiso que deben asumir los investigadores, tanto chilenos como extranjeros con museos $\mathrm{u}$ otras instituciones que posean colecciones, de 
enviar ejemplares a los respectivos países desde donde los ejemplares fueron colectados. De igual modo se hace necesario llevar a cabo estudios para conocer la presencia o ausencia de especies en aquellas áreas geográficas de Chile donde a la fecha no existen registros de bivalvos dulceacuícolas que permita a futuro elaborar una propuesta de clasificación de las aguas continentales chilenas.

\section{AGRADECIMIENTOS}

Los autores agradecen muy sinceramente al Dr. Cristián F. Ituarte, Director del Departamento de Zoología de Invertebrados del Museo de La Plata, Buenos Aires, Argentina, por todo el apoyo prestado para la confección del documento. A nuestros colegas Cristian Sobarzo, del Departamento de Ciencias Biológicas y Químicas, de la Universidad Católica de Temuco, por todas las diligencias llevadas a cabo en el Museo de La Plata, Argentina y Gladys Lara por facilitar los especímenes provenientes de Río Negro, Istmo de Ofqui. Proyecto financiado por la Dirección de Investigación de la Universidad Católica de Temuco (Proyecto DIUCT 2000-4-01).

\section{LITERATURA CITADA}

BAKER FC (1929) On the division of the Sphaeriidae into two subfamilies and the description of a new genus of Unionidae, with descriptions of new varieties. American Midland Naturalist 10: 220-223.

BURCH JB (1975) Freshwater sphaeriacean clams (Mollusca: Pelecypoda) of North America. Malacological Publications, The University of Michigan, Ann Arbor, Michigan. xi +95 pp.

BONETTO AA (1961) Investigaciones acerca de las formas larvales en el género Diplodon y su aplicación a los estudios sistemáticos. Dirección General de Recursos Naturales, Ministerio de Agricultura, Provincia de Santa Fe, Santa Fe, República Argentina. 48 pp.

BONETTO AA (1973a) Náyades de la Patagonia. Revista de la Asociación de Ciencias Naturales del Litoral 4: 177- 185.

BONETTO AA (1973b) Estudio revisivo de las colecciones de náyades de D'Orbigny existentes en el Museo Británico. Sociedad Científica Argentina Sección Santa Fe 1: 17-25.

BONETTO AA, M TASSARA \& A RUMI (1986) Australis n. subgen. de Diplodon Spix (Bivalvia, Unionacea) y posibles relaciones con Hyriidae australianos. Boletín de la Sociedad de Biología de Concepción (Chile) 57: 55-61.

BOWDEN J \& D HEPPELL (1968) Revised list of British Mollusca. 2. Unionacea-Cardiacea. Journal of Conchology London 26: 237-272.
CLARKE AH (1973) The freshwater molluses of the Canadian interior basin. Malacologia 13: 1-509.

CLARKE AH (1981) The freshwater molluscs of Canada. Ottawa National Museum of Natural Sciences, National Museum of Canada, Ottawa, Canada. 446 pp.

DAVIS GM \& M MULVEY (1993) Species status of Mill Creek Elliptio. Savannah River Site, National Environmental Research Program, Publication SRONERP 22, Aiken, South Carolina. 55 pp.

DILLEHAY TD (1984) A late ice-age settlement in southern Chile. Scientific American 251: 100-109.

D'ORBIGNY AD (1846) Voyage dans l'Amerique Méridionale exécuté pendant les années 1826-1833, volume 5, part. 3: Mollusques. P. Bertrand, Paris, Francia. $758 \mathrm{pp}$.

ELLIS AE (1978) British freshwater bivalve Mollusca: Keys and notes for the identification of the species. Academic Press, London, United Kingdom. 109 pp.

FIGUEIRAS A (1965) La malacofauna dulceacuícola del Uruguay. Parte II: Pelecipoda. Comunicaciones de la Sociedad Malacológica del Uruguay 1: 223-270.

GRAF DL \& D Ó FOIGHIL (2000) Molecular phylogenetic analysis of 28S rDNA supports a Gondwanan origin for Australasian Hyriidae (Mollusca: Bivalvia: Unionoida). Vie et Milieu 50: 245-254.

GRAY JE (1847) A list of the genera of recent Mollusca, their synonymy and types. Proceedings of the Zoological Society of London 15: 129-219.

GRAY JE (1855) List of the shells of South America in the collection of the British Museum, collected and described by M. Alcide d'Orbigny, in the "Voyage dans l'Ámerique Méridionale", Taylor \& Francis, London, United Kingdom. 89 pp.

HAAS F (1930-1931) Versuch einer kritischen sichtung der südamerikanischen Najaden. Senckenbergiana 12: 175; 13: $30 ; 13: 87$

HAAS F (1969) Superfamilia Unionacea. Das Tierreich (Berlin) Lieferung 88, Seite I-X: 1-663.

HEARD WH (1965) Comparative life histories of north american pill clams (Sphaeriidae: Pisidium). Malacologia 2: 381-411

ITUARTE CF (1995) Nuevos registros de Pisidium Pfeiffer, 1821 y Sphaerium Scopoli, 1777 (Bivalvia: Sphaeriidae) en Chile, Bolivia y noroeste argentino. Neotropica 41: 31-41.

ITUARTE CF (1996) Argentine species of Pisidium Pfeiffer, 1821, and Musculium Link, 1807 (Bivalvia: Sphaeriidae). The Veliger 39: 189-203.

ITUARTE CF (1999) Pisidium chilense (d'Orbigny, 1846) and new species of Pisidium C. Pfeiffer, 1821 from southern Chile (Bivalvia, Sphaeriidae). Zoosystema 21: 249-257.

ITUARTE CF \& S GORDILLO (1991) Nuevas citas de pelecípodos dulceacuícolas de Isla Gable, Tierra del Fuego, Argentina. Neotropica 37: 29-30.

JARA-SEGUEL P, S PEREDO, C PALMA-ROJAS, E PARADA \& G LARA (2000) Quantitative karyotype of Diplodon chilensis (Gray 1828) (Bivalvia: Hyriidae). Gayana Zoología (Chile) 64: 189 -193.

KEEN M \& P DANCE (1969) Family Corbiculidae. En Moore RC (ed) Treatise on invertebrate paleontology, Parte N, Volumen 2, Mollusca 6, Bivalvia: 669-670. Geological Society of America y Kansas University Press, Lawrence, Kansas. 
KORNIUSHIN AV (1996) Growth and development of the outer demibranch in freshwater clams (Mollusca, Bivalvia): a comparative study. Annales Zoologici Fennici 46: 111-124.

KORNIUSHIN AV (1997) Patterns of gills structure and development as taxonomic characters in bivalves molluscs (Mollusca, Bivalvia). Annales Zoologici Fennici 46: 245-254.

KORNIUSHIN AV (1998) Notes on the anatomy of some species of Sphaerium sp. (Mollusca, Bivalvia) from tropical regions with revision of their taxonomic status. Vestnik Zoologii 32: 3-12.

KORNIUSHIN AV (2000) Review of the family Sphaeriidae (Mollusca: Bivalvia) of Australia, with the description of four new species. Records of the Australian Museum 52: 41-102.

KUIPER JGJ (1983) The Sphaeriidae of Australia. Basteria 47: 3-52

KUIPER JGJ \& W HINZ (1983) Zur fauna der kleinmuscheln in der anden (Bivalvia: Sphaeriidae). Archiv für Molluskenkunde 114: 109-116.

KUSTER HC (1842-1862) Die Flussperlmuscheln (Unio et Hyria). En: Martini FHW \& JH Chemnitz (eds) Abbildungen nach der Natur, Systematisches Conchylien Cabinett 9:2. Martini \& Chemnitz, London, United Kingdom.

LOZADA E \& C OSORIO (1995) Mollusca. En: Simonetti JA, MTK Arroyo, A Spotorno \& E Lozada (eds) Diversidad biológica de Chile: 148-155. Comité Nacional de Diversidad Biológica, Comisión Nacional de Investigación Científica y Tecnológica, Santiago, Chile.

MACKIE GL (1979) Dispersal mechanism in Sphaeriidae. Bulletin of the American Malacological Union 1979: 17-21.

MEIER-BROOK C (1970) Untersuchungen zur biologie einiger Pisidium Arte. Archiv für Hydrobiologie, Supplement (Germany) 38: 73-150.

MORGAN G (1982) Historical and ecological factors in the evolution, adaptive radiation and biogeography of freshwater mollusks. American Zoologist 22: 375395.

MORTON B (1985) The reproductive strategy of the mangrove bivalve Polymesoda (Geloina) erosa (Bivalvia: Corbiculoidea) in Hong Kong. Malacological Review 18: 83-89.

OJEDA FP (1998) Estado actual de la conservación de la biodiversidad biológica en Chile. Revista Chilena de Historia Natural 71: 117-120.

OSORIO C \& N BAHAMONDE (1970) Lista preliminar de lamelibranquios de Chile. Boletín del Museo Nacional de Historia Natural (Chile) 31: 187-256.

PARADA E \& S PEREDO (1994) Un enfoque ecológico evolutivo de las estrategias de historia de vida de los híridos chilenos (Mollusca, Bivalvia). Boletín de la Sociedad de Biología de Concepción (Chile) 65: 7180.

PARADA E, S PEREDO, G LARA \& F ANTONIN (1989a) Contribución al conocimiento de los Hyriidae chilenos. Boletín de la Sociedad de Biología de Concepción (Chile) 60: 173-182.
PARADA E, S PEREDO, G LARA \& I VALDEBENITO (1989b) Growth, age and life span of the freshwater mussel Diplodon chilensis chilensis (Gray, 1828). Archiv für Hydrobiologie (Germany) 115: 563-573

PARADA E, S PEREDO \& C GALLARDO (1990) Tácticas reproductivas y dinámica poblacional de Diplodon chilensis (Gray, 1828) (Bivalvia: Hyriidae). Revista Chilena de Historia Natural 63: 23-35.

PARK JK \& D Ó FOIGHIL (2000) Sphaeriid and Corbiculid clams represent separate heterodont bivalve radiations into freshwater environments. Molecular Phylogenetics and Evolution 14: 75-88.

PARODIZ JJ (1977) Mollusca. En: Hulbert SH (ed) Biota acuática de Sudamérica austral: 320-329. San Diego State University, San Diego, California.

PARODIZ JJ \& AA BONETTO (1963) Taxonomy and zoogeographic relationships of the South American naiades. Malacologia 1: 179-213.

PEREDO S \& E PARADA (1984) Gonadal organization and gametogenesis in the fresh-water mussel Diplodon chilensis chilensis. The Veliger 27: 126-133.

PEREDO S \& E PARADA (1986) Reproductive cycle in the freshwater mussel Diplodon chilensis chilensis (Mollusca: Bivalvia). The Veliger 28: 418-425.

PHILIPPI RA (1847-1848) Abbildungen und beschreibungen neuer oder wenig gekanntes conchylien. Kassel 2: 1847: 153-231; 3: 1847: 1-10; 1848: 11-82.

PHILIPPI RA (1869) Bemerkungen uber die chilenischen unionen. Malakozoologishe Blätter 1: 43-49.

REES WJ (1965) The aerial dispersal of Mollusca. Proceedings of the Malacological Society of London 36: 269-282

SÁNCHEZ M \& J INOSTROZA (1985) Excavaciones arqueológicas en el alero Quino 1. Boletín del Museo Regional de La Araucanía (Chile) 2: 53-62.

SIMPSON CT (1914) A descriptive catalogue of the naiades or pearly freshwater mussels. Detroit (Bryant Water) ixi: $1-1540$.

SOBARZO C, P JARA-SEGUEL, S PEREDO \& E PARADA (en prensa) Primer registro de Musculium argentinum (d'Orbigny 1835) (Bivalvia: Sphaeriidae) en aguas continentales chilenas. Gayana Zoología (Chi1e).

SOWERBY GB (1864-69) Monograph of the genera Unio, Mycetopus, Anodon, Castalia. Conchological Iconica XVI-XVII: $16-17$.

STAROBOGATOV YI (1977) Mollusques bivalves. En: Kutikova LA \& YI Starobogatov (eds) Guide to the identification of non-marine invertebrates of the Europeans part of the URSS: 123-151, Zoological Institute, Russian Academic of Science, Leningrad, Russia.

VALDOVINOS C (1999) Biodiversidad de moluscos chilenos: base de datos taxonómica y distribucional. Gayana Zoología (Chile) 63: 111-164.

WILLIAMS JD \& M MULVEY (1997) Recognition of freshwater mussel taxa. A conservation challenge. En: Meffe GK \& R Carroll (eds) Principles of conservation biology: 64-65. Sinauer Associates, Inc. Publishers, Sunderland, Massachusetts. 